\title{
Influence of Heat-source Temperature on Convection- based Tilt Sensor Output
}

\author{
Yong-hong Zheng \\ College of Science \\ Beijing Information Sci. and Tech. University \\ Beijing, China \\ E-mail: hankzyh@126.com
}

\begin{abstract}
According to the sensitive mechanism of convectionbased tilt sensor, temperature field plays a key role in the sensing tilt. The tilt sensor output has one to one correspondence with the temperature field, which is variety with heat-source temperature. Using ANSYS, temperature flow fields are simulated by variety heat-source temperatures in a two dimension hermetic chamber, the differences of temperature between hotwires are obtained, the outputs based on these are calculated, also compared with measured values. From the results the output relation with the heat-source temperature is given qualitatively1.【医】 figurate; qualltative, through this relation the output of sensor can be improved, meanwhile output drifted with heat-source temperature can be decreased by provided circuit.
\end{abstract}

Keywords- heat-source; convection-based; difference of temperature; tilt

\section{INTRODUCTION}

In 1990's, convection-based tilt sensor had been found broad application as in [1-4]. Because the mass of gas is small and there is no proof mass, the tilt sensor has its characteristics of resisting oscillation and shock. At present, convection-based tilt sensor can be seen in many fields like military, spaceflight, aviation, civil and so on.

Convection-based tilt sensor is made according to pendulum characteristics of the natural convection of gas. Sensitive mechanism of gas convection-based tilt sensor is shown in Figure. 1. A heat-source and two hotwires are placed in a hermetic chamber; a wire like heat-source is placed along axis of the chamber, hotwires are placed symmetrically and compose the arms of bridge circuit. Yet temperature distribution induced by the heat source plays a key role in the sensing tilt as in [5]. When the sensor is in horizontal state two hotwires sense the same temperature of fluid flow, their resistances are equal. The difference of temperature between hotwires is zero. The output of bridge circuit is zero. When sensor is in tilt state two hotwires sense the different temperature of the flow, the corresponding resistances are not equal to each other. The difference of temperature between hotwires is variety with the tilt. The bridge outputs a voltage signal corresponding to the angle.

\author{
Shi-liu Peng \\ State Key Laboratory of High-temperature Gas Dynamics \\ Institute of Mechanics, CAS \\ Beijing, China \\ E-mail: slpeng@hotmail.com
}

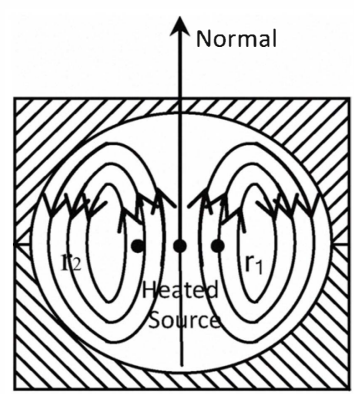

(a) Horizontal state

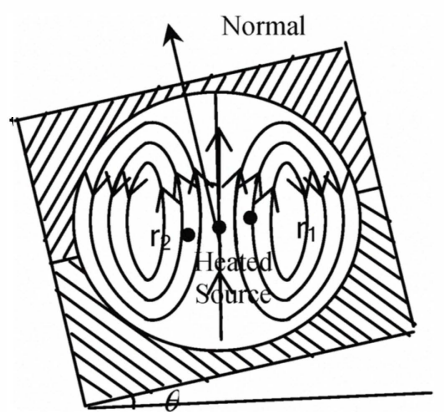

(b) Tilt state

Figure 1. Schematic diagram of gas pendulum

II. The Detecting Circuit

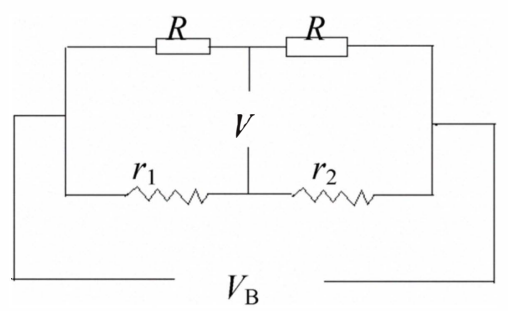

Figure 2. Bridge circuit

Bridge circuit is showed in Fig.2. The output of bridge circuit is given as in Zheng, Y.H. [6].

$$
V=V_{B} \frac{r_{1}-r_{2}}{2\left(r_{1}+r_{2}\right)}
$$

where $r_{1}$ and $r_{2}$ are the resistance of hotwires, $R$ is accurate resistance, $V_{\mathrm{B}}$ is power supply volt of bridge, $V$ is output of bridge. As sensor is in tilt state, the resistance of one hotwire increases, the other must decrease, and increased value is approximate to decreased value, $r_{1}-r_{2}$ is constant basically. Let $r=r_{1}-r_{2}$, then the bridge output is written by

$$
V=V_{B} \frac{r_{1}-r_{2}}{2 r}
$$

Variety of resistance with temperature is 


$$
r(T)=r_{0}\left[1-a_{1}\left(T-T_{0}\right)\right]
$$

where $a_{1}=3.90802 \times 10^{-3}{ }^{0} \mathrm{C}^{-1}, r_{0}$ is resistance of reference temperature $T_{0}$, referenced to Sheng, S.Z., Xu, Y.T. and Yuan, H.J. [7]

By the expression of (3), $r_{1}$ and $r_{2}$ are written as

$$
\begin{aligned}
& r_{1}(T)=r_{10}\left[1 \dashv a_{1}\left(T-T_{1}\right)\right] \\
& r_{2}(T)=r_{20}\left[1 \dashv a_{1}\left(T-T_{2}\right)\right]
\end{aligned}
$$

where $T_{1}$ and $T_{2}$ are the temperature of $r_{1}$ and $r_{2}$. Hotwires are same long, therefore $r_{10}=r_{20}$. Let $r_{0}=r_{10}=r_{20}$, a relation can be gotten

$$
\Delta r=r_{2}-r_{1}=r_{0} \alpha_{1}\left(T_{2}-T_{1}\right) \approx r_{0}\left[4 \times 10^{-3}\left(T_{2}-T_{1}\right)\right]
$$

By (1) and (6), another relation can be gotten again

$$
V=\frac{V_{B}}{2 r} \alpha_{1} r_{0}\left(T_{1}-T_{2}\right)
$$

The difference of temperature between hotwires determines bridge output due to the expression of (7). If heat-source temperature changes, difference of temperature between hotwires will change too. The test of tilt is altered under the variety heat-source temperature. The property of convectionbased tilt sensors is changed, that is sensitivity drifted with temperature.

\section{NUMERICAL SimUlation OF FLOW IN THE HERMETIC CHAMBER}

It is convenient to use software for analysis in details; the software of ANSYS is used to get numerical solutions as in [8].

\section{A. Modeling}

1) Establish a Title and Preferences Choose FLOTRAN CFD analysis.

2) Define Element Types Choose 2D FLUID141.

3) Create Areas

Choose round represents hermetic chamber.

\section{4) Define Lines, Mesh, and Plot Elements}

Set the number of element division to 625 .

\section{B. Loading}

\section{1) Apply Velocity Boundary Conditions}

Set velocity of nodes on outside edge are zero along $\mathrm{x}$ and $\mathrm{y}$ direction.

\section{2) Apply Thermal Boundary Conditions}

Set temperature of nodes on outside edge are $293 \mathrm{~K}$, temperature of heat source is $303 \mathrm{~K}, 323 \mathrm{~K}, 343 \mathrm{~K}, 373 \mathrm{~K}$.

3) Set C Solution Options and Execution Controls Set the field to thermal, EXEC to 200, RFL File Overwrite Freq field to 50 .

Set the Output summary frequency field to 50 .

\section{4) Set Fluid Properties}

Set the Density, Viscosity, Conductivity and Specific heat fields to AIR-SI. Allow density variations.

\section{5) Set FLOTRAN Environment Parameters}

Set the Acceleration in y direction field to 9.8 .

\section{Solve the Problem}

Choose TDMA. Make sure that the value of the No. of TDMA sweeps for pressure field is 100 .

Now keeping environment temperature is $20^{\circ} \mathrm{C}$, heat-source temperature (HT) is changed. In Figure. 3 is the temperature distribution with different heat-source temperature as tilt of sensor is 20. From Fig3, it is very clear that these four temperature fields are different. The isometric lines of temperature forming circles are concentric shown in Fig.3 (a), which indicates the gradient of temperature field is little. The isometric lines of temperature forming circles move above gradually shown in Fig.3 (d), which indicates the gradient of temperature field is large. There are dissimilar temperature distributions at different heat-source temperature, while the temperature is high, and the gradient of temperature along radial direction is apparently large. Using the same way temperature fields are simulated at different sensor tilt.

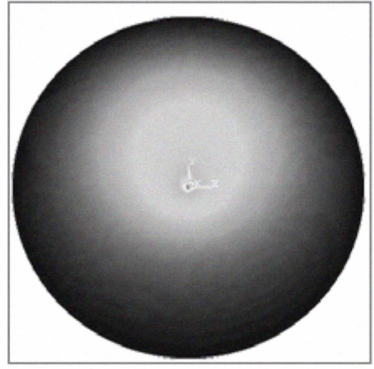

(a) $\mathrm{HT}$ is $30^{\circ} \mathrm{C}$

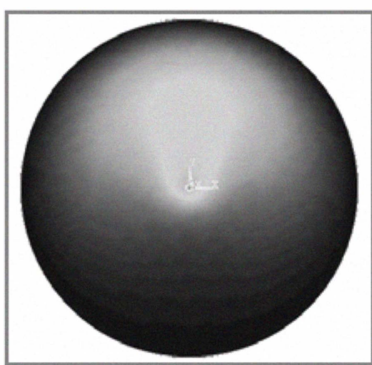

(c) $\mathrm{HT}$ is $70^{\circ} \mathrm{C}$

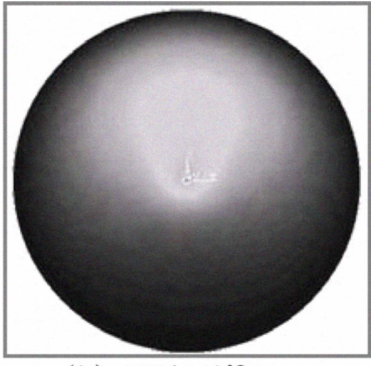

(b) $\mathrm{HT}$ is $50^{\circ} \mathrm{C}$

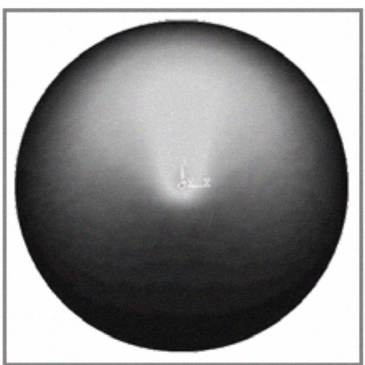

(d) $\mathrm{HT}$ is $100^{\circ} \mathrm{C}$
Figure 3. Temperature field diagram

From simulated temperature fields, the differences of two hotwires can be calculated at the variety temperatures of heat-source. The table 1 gave separately the differences of two hotwires as sensor tilt is $20^{\circ}, 30^{\circ}$ and $45^{\circ}$. 
TABLE I. THE RELATIONSHIP BETWEEN HEAT-SOURCE AND THE DIFFERENCE OF HOTWIRES

\begin{tabular}{|c|c|c|c|}
\hline \multirow{2}{*}{$\begin{array}{l}\text { Heat-source } \\
\text { temperature } \\
\left({ }^{\circ} \mathrm{C}\right)\end{array}$} & \multicolumn{3}{|c|}{ The difference of hotwires $\left({ }^{\circ} \mathrm{C}\right)$} \\
\hline & Sensor tilt $20^{\circ}$ & Sensor tilt $30^{\circ}$ & Sensor tilt $\mathbf{4 5}^{\circ}$ \\
\hline 30 & 0.47 & 1.84 & 3.49 \\
\hline 40 & 1.23 & 2.47 & 4.12 \\
\hline 50 & 1.96 & 3.01 & 4.78 \\
\hline 60 & 2.61 & 3.67 & 5.33 \\
\hline 70 & 3.21 & 4.26 & 5.91 \\
\hline 80 & 3.82 & 4.87 & 6.40 \\
\hline 90 & 4.40 & 5.62 & 7.02 \\
\hline 100 & 4.85 & 6.14 & 7.43 \\
\hline 110 & 5.37 & 6.63 & 8.03 \\
\hline 120 & 5.79 & 7.11 & 8.31 \\
\hline
\end{tabular}

It is obvious that the more heat-source temperature is raised the more difference of hotwires is gotten. In accordance with the difference of hotwires and the expression of (7), the outputs of sensor are calculated with variety temperatures of heatsource when tilts are $20^{\circ} 、 30^{\circ}$ and $45^{\circ}$. Furthermore the above results are compared with the values of experiment. Figue. 4 shows the final results.

The output of sensor changed with the heat-source temperature even at the same tilt, which means the sensitivity of sensor drifted with heat-source temperature. When heatsource temperature is low, the output voltage is large; when environment temperature is high, the output voltage is small. The output voltage is proportion to the heat-source temperature For improving the sensitivity of sensor, the heat-source temperature is raised as far as possible.

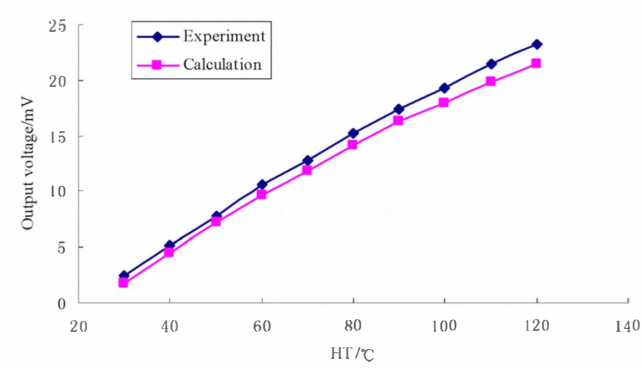

(a) Inclination angle is $20^{\circ}$

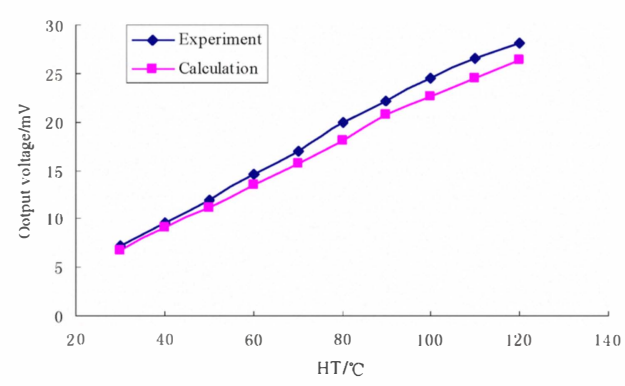

(b) Inclination angle is $30^{\circ}$

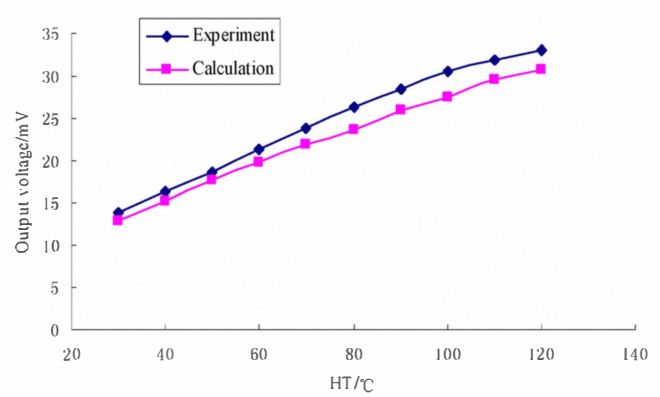

(c) Inclination angle is $45^{\circ}$

Figure 4. Outputs with different HT

On the other hand if temperature field would be kept invariant, output of sensor is not changed because of the heatsource temperature unchanged, as in [9]. Circuit can be designed for keeping the heat-source temperature invariant. The circuit is drawn in Figure.5. $R$ is a resistance of bridge, $R_{\mathrm{b}}$ is adjustable resistance of the same bridge.

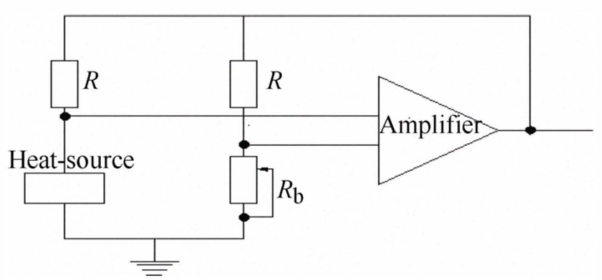

Figure 5. Feedback circuit of heat-source

\section{CONCLUSIONS}

According to the numerical simulation and the experiment, the output voltage of sensor is proportion to the heat-source temperature. The more the temperature is raised, the more the output is gotten. So the sensitivity of sensor is high.

Through raising the heat-source temperature, the sensitivity of sensor is improved. Not only is the temperature restricted by the power of electrical source, but also approximation of boundary conditions of flow in hermetic chamber is not always right as in [10], and these confined the increase of the temperature. 
Using the results above, circuit can be designed for keeping the heat-source temperature invariant. The output voltage of bridge is unchanged, so the sensitivity of sensor is unchanged.

\section{REFERENCES}

[1] Zhang Fu-xue. Gas Pendulum Acceleration Sensor[J]. Chinese Journal of Electronics, 2000, 9(1): 14-16.

[2] DAUDERSTADT U A, SARRO P M,FRENCH P J. Temperature Dependence And Drift of a Thermal Accelerometer[J], Sens,Actuators, 1998, A66:244-249.

[3] DAO R, MORGAN D E, KRIES H H, et al. Convective Accelerometer And Inclinometer:US,5581034[P].1996.

[4] Zhang Fu-xue, Chen Zhan-xian, Luo Yu-hua. Gas Pendulum Tilt Sensor. China CN1064351[P].1992.
[5] Zheng, Y.H., " Analysis of stream field of Gas Pendulum Tilt Sensor" [J]. Micronanoelectronic Technology2007, 7/8 242-244.

[6] Zheng, Y.H., Peng, S.L. "A Method of Eliminating Influence of Environmental Temperature on Tilt Sensor Property" Piezoelectrics\&Acoustooptics, vol, 30 (1) , 2008, pp. 33-35.

[7] Sheng,S.Z.,Xu,Y.T.,and Yuan,H.J., Hot Wire Anemometry, China Science Technology Press , Beijing, 2003.

[8] Li, L.M., ANSYS Finite Element Analysis practicality Course of Study, Tsinghua University Press, Beijing, 2002.

[9] Zheng, Y.H., Peng, S.L. "Application of Flow Similarity to Convectionbased Tilt Sensor," Journal of University of Electironic Science and Technology of China. 2007,36 (5): :931-934.

[10] Zheng, Y.H., Peng, S.L. "The Dependence of Sensor Property on Flow in Hermetic Chamber of the Gas Pendulum Tilt Sensor", CMCE. 2010.84-87. 